\section{MS39-P20 Designing a powder diffraction instrument optimised for hydrogenous materials. HOD - the Hydrogen Observation Diffractometer concept for ESS}

Paul F. Henry ${ }^{1,2}$, Mark T. Weller ${ }^{3}$, Chick C. Wilson ${ }^{3}$, Paul R. Raithby $^{3}$, Holger Kohlmann ${ }^{4}$, Sten Eriksson ${ }^{2}$, Chris Knee ${ }^{2}$

1. European Spallation Source (ESS) AB, PO Box 176, Lund, Sweden

2. Chemical Engineering Dept., Chalmers University of Technology, Gothenburg, Sweden

3. Department of Chemistry, University of Bath, Bath, UK

4. University of Leipzig, Leipzig, Germany

email: paul.henry@esss.se

Hydrogen is the most important element on Earth and has arguably, over recent years, become even more important in the context of for the Grand Challenges facing Humankind; it is pervasive and unique in materials science, chemistry and biological systems. In addition to its strong structure and property directing role, it has itself become an increasingly important "guest" in materials, for example in relation to its huge potential in energy storage and energy vector applications. Therefore, understanding both the fundamental and applied behaviour and function of hydrogen - its location and its motion - in determining the properties of a system, will lie at the core of the majority of technological advances of the next few decades.

The problem facing the experimentalist is that hydrogen is a very weak $\mathrm{X}$-ray scatterer and, even with the brightest synchrotron sources, it is almost impossible to accurately determine hydrogen positions. Neutron scattering potentially offers the solution, but is complicated by the high incoherent scattering cross section of $1 \mathrm{H}$, which gives very high backgrounds. While deuteration is often used, the positive / negative scattering contrast of $\mathrm{D} / 1 \mathrm{H}$ becomes an issue if deuteration is incomplete, it is expensive or impossible to deuterate materials and deuteration often changes the physical properties of the material. Some progress has been made in this area over the last several years and techniques are under development that can significantly reduce and even control the scattering properties of hydrogen in neutron scattering.

However, no current generation instrument can deliver these to the experimentalist. The ESS, currently in the initial stages of construction in Lund, Sweden is a new type of spallation source, using a long pulse. This offers new possibilities for instrument design combining the features from reactor-based, continuous sources with time-of-flight energy sensitivity afforded by the pulsed nature of the source. HOD - the hydrogen observation diffractometer is the result of several years of development work aimed at delivering an optimsed instrument for hydrogenous materials without the need to deuterate.

Keywords: powder, neutron, hydrogen, diffractometer
MS39-P21 How to determine the space group of a twinned crystal or one with metric specialization

\author{
Howard D. Flack ${ }^{1}$
}

1. Chimie minérale, analytique et appliquée, University of Geneva, Switzerland

email: crystal@flack.ch

The excellent text Determination of Space Group by Looijenga-Vos \& Buerger in the $5^{\text {th }}$ edition of International Tables for Crystallography Volume A Space-group symmetry is dated. It will be replaced in the $6^{\text {th }}$ edition by the text Methods of space-group determination by Shmueli, Flack \& Spence. A major limitation of both of these texts is that the tables of values only apply to single-crystal samples. Using established theory available in the literature, tables of values applicable to many twinned crystals and many cases of metric specialization have been produced. The tables are arranged by observed Bravais-lattice type. For each observed Bravais-lattice type, the values are arranged horizontally by Laue and crystal class, and vertically by reflection conditions. The tables indicate those space groups obeying the observed Bravais-lattice type, the observed Laue and crystal classes, and the observed reflection conditions. The tables have been implemented in a commercially-available spreadsheet on a pc and will be available for demonstration.

Keywords: space group, twin, metric specialization 